\title{
Large Area 3D Structural Characterization by Serial Sectioning Using Broad Ion Beam Argon Ion Milling
}

\author{
P. Nowakowski ${ }^{1}$, M.L. Ray ${ }^{1}$ and P.E. Fischione ${ }^{1}$ \\ ${ }^{1 .}$ E. A. Fischione Instruments, Inc., 9003 Corporate Circle, Export, PA, USA
}

Structural characterizations are typically two dimensional (2D) plan views cut through a sample. While 2D studies are accurate and yield good results, there are instances where three dimensional (3D) characterization of sample volume is required to fully understand the material's properties or structural behavior. Examples include habit plane and the orientation relationship between phase determination in bi-phase or multiphase materials [1], fatigue crack propagation [2, 3], and statistical grain size and shape measurements. Moreover, only 3D volume electron backscatter diffraction (EBSD) with five-parameter grain boundary analysis can provide a full understanding of grain boundaries roles and influences on a material's properties [4]. The most common technique used for 3D volume serial sectioning in focused ion beam milling (FIB). This technique yields accurate results; however, it has limitations:

- the observed volume dimension is relatively small: 50 x 50 x $50 \mu \mathrm{m}^{3}$ for Ga FIB [3] and $150 \times 120 \mathrm{x}$ $80 \mu \mathrm{m}^{3}$ for Xe plasma FIB [5]

- the resulting FIB material damage to the sample requires damage removal steps prior to EBSD acquisition [6].

Lin [7] achieved a 3D EBSD volume of $150 \times 100 \times 80 \mu \mathrm{m}$ by combining mechanical polishing and electropolishing to obtain a surface quality suitable for EBSD acquisition.

In this work, we show a recent development of large 3D volume serial sectioning using broad ion beam (BIB) argon ion milling. The stability and repeatability of the Model 1061 SEM Mill [Fischione Instruments] argon ion sources allow high quality, large volume sectioning of up to $500 \mathrm{x} 400 \mathrm{x}$ $100 \mu \mathrm{m}^{3}$ (Figure 1). Ion milling also results in a sample surface suitable for high-speed EBSD acquisition without the need for damage removal steps, even in multiphase materials with significant differences in hardness and milling rates $[8,9]$. The BIB serial section slicing of Al samples $(5 \mu \mathrm{m}$ thick slices and $5 \mu \mathrm{m}$ EBSD mapping step size) allow grain size, shape, and intra-grain misorientation analyses (Figure 2). The application of this technique to multiphase materials and grain boundary network investigation will be also discussed.

References:

[1] S Zaefferer and SI Wright in "Electron backscatter diffraction in materials science", eds. AJ Schwartz et al, (Springer, Boston), p. 109.

[2] S Birosca et al, Acta Mater. 59 (2011), p. 1510.

[3] S Zaefferer, SI Wright, and D Raabe, Metall. Mater. Trans. 39 (2008), p. 374.

[4] A Khorashadizadeh et al, Adv. Eng. Mater. 13 (2011), p. 237.

[5] TL Burnett et al, Ultramicroscopy 161 (2016), p. 119.

[6] N Saowadee, K Agersted, and JR Bowen, Jour. Microsc. (Oxford, U.K.) 246 (2012), p. 279.

[7] FX Lin et al, Mat. Charact. 61 (2010), p. 1203.

[8] P Nowakowski et al, Microsc. Microanal. 22 (2016), p. 12.

[9] P Nowakowski et al, European Microscopy Congress Proceedings (2016), p. 1082. 

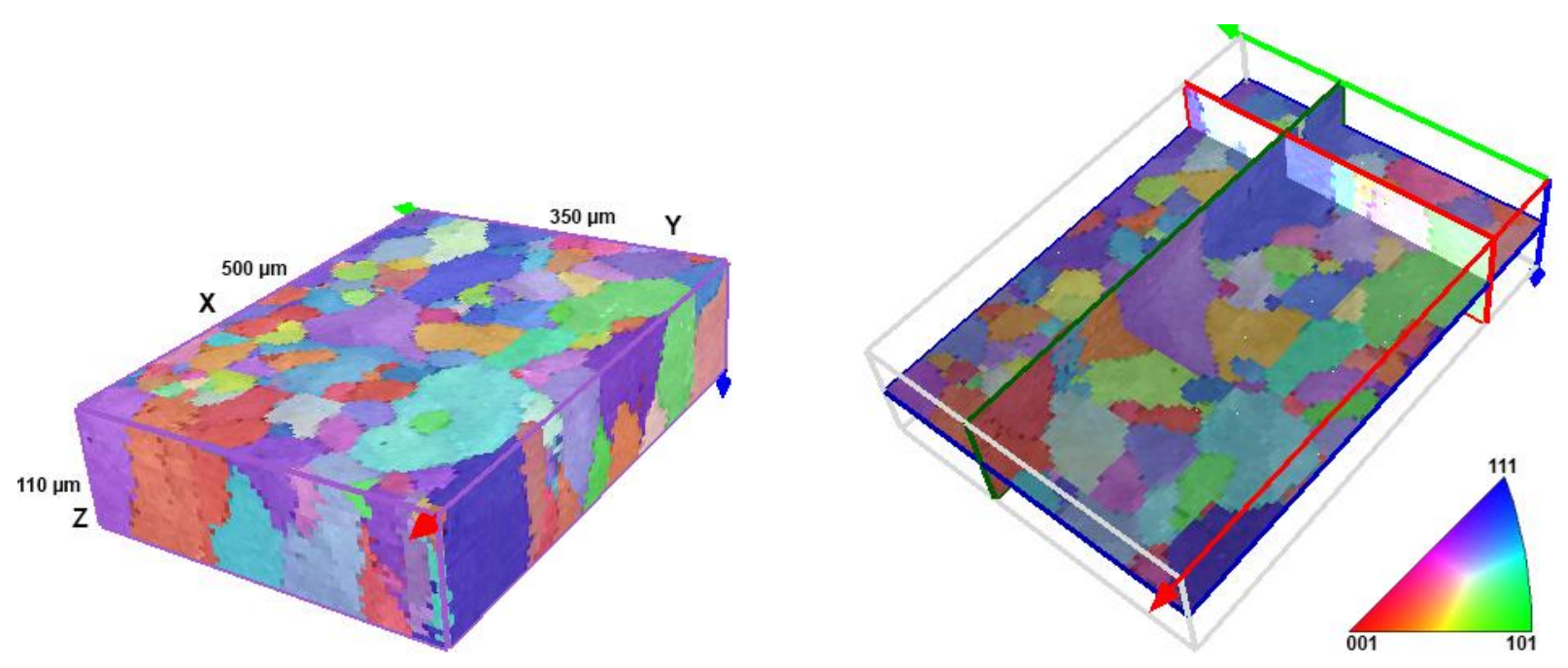

Figure 1. Pure aluminum 3D reconstructed volume, $500 \times 350 \times 110 \mu \mathrm{m}(5 \mu \mathrm{m}$ thick slices). EBSD data acquired at $20 \mathrm{kV}$ using $8 \times 8$ camera binning: 3D perspective (left); 3D section view in $\mathrm{XY}, \mathrm{XZ}$, and $\mathrm{YZ}$ plans (right). Inverse pole figure (IPF) in X direction in color-coded EBSD maps.
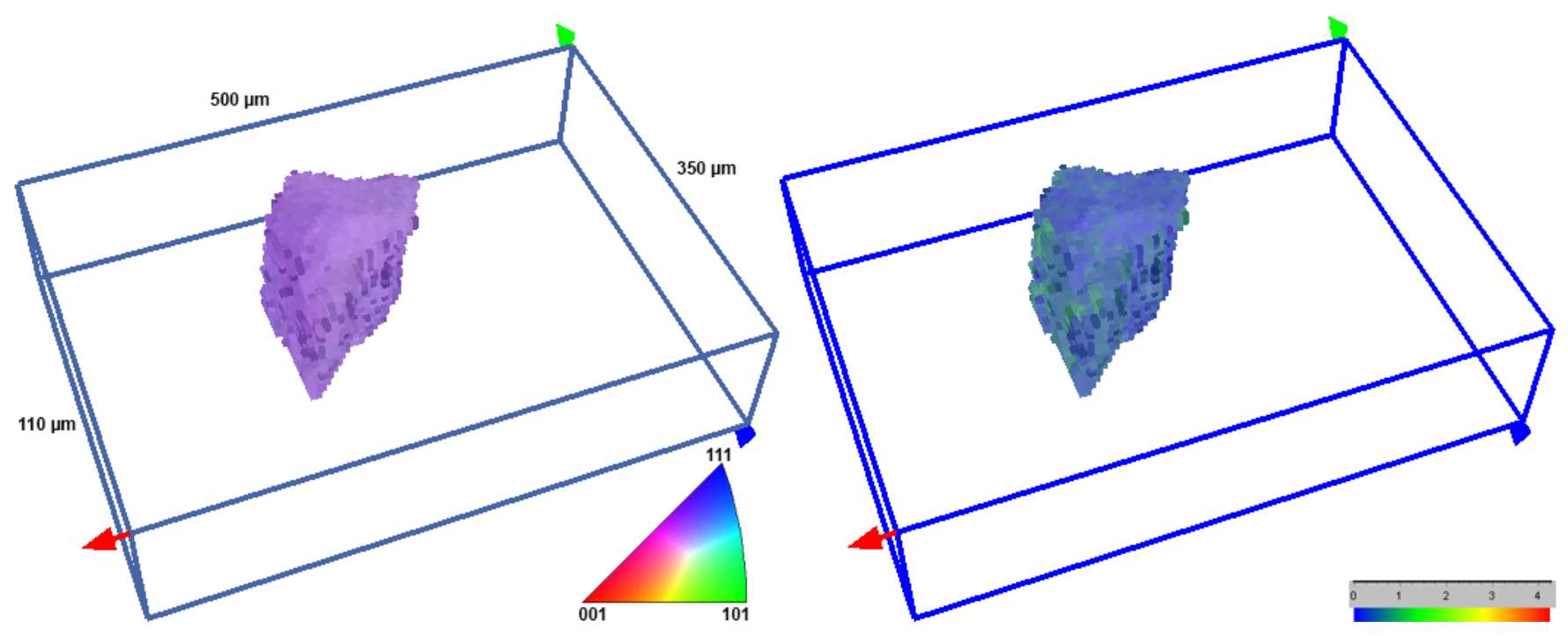

Figure 2. Large Al grain isolated after grains detection: IPF in X direction, 3D EBSD map (left), local misorientation (right), 3D EBSD map. 